AC 2010-402: VIRTUAL LABORATORY FOR STUDY OF THE ELECTRIC MACHINES PARAMETERS AND CHARACTERISTICS

Radian Belu, Drexel University 


\title{
Virtual Laboratory for Study of the Electric Machines Parameters and Characteristics
}

\begin{abstract}
Computing and communication technology have had a significant impact on engineering education. This technology has significantly improved online, distance, collaborative learning, as well as the use of the virtual experiments and simulations in engineering education. One of the distinguishing features of engineering education is that the laboratory work is an integral part and its purpose is bringing the students closer to real situations that they may encounter. Advances in electric machinery, control, and instrumentation systems and interfaces call for a continuous upgrading of facilities that will support energy conversion education. Costs to equip an energy conversion laboratory for teaching undergraduates could easily run to a hundred thousand dollars. These prohibitive costs have forced many electrical engineering programs to trade off good quality laboratory equipment for affordable and significantly less accurate scaled-down models, thus, usually exhibiting characteristics that are far from what is considered typical. Moreover, the typical traditional electric machines laboratories offer very limited access to equipment and instrumentation. The shortcomings of traditional laboratories include among others: cost, safety, limited students' access and space requirements. In this context virtual laboratories and experiments have become an appealing alternative to the traditional settings. Three key problems are discussed here: the purpose, the content and the impact of such a laboratory in studying and learning electrical machines.
\end{abstract}

\section{Introduction}

Engineering education can reach the desired level if practical and laboratory works are taught together with theory. Laboratories are common elements of training for the students in order to gain experience and to acquire knowledge required in present day industry. Practical experiments in a laboratory helps students gain practical skills, become familiar with the instrumentation and measurement systems, and prepare for professional life. The conventional way of providing practical experience to engineering students is through the use of extensive laboratory-based systems. Such systems require an actual hardware setup and a set of laboratory measurement systems and instrumentation that are often very costly to build or upgrade, and difficult to maintain. For safety and security reasons, access to laboratorybase systems is usually limited to a certain time and can only be given in the presence of a local facilitator. However, as education and technology merge the opportunities for teaching and learning have expanded at the very rapid rate of change in the field of technology poses special problems for academic institutions. There is a continual need to update and augment the content of courses and laboratories to keep pace with this change, especially in the area of engineering education. The central problem still remains the same; providing for students meaningful and relevant practical experiences while being limited by very finite resources in the provision of laboratory infrastructure. One solution to this problem is to use computers with suitable front-end design to simulate experiments. Furthermore, information and experience sharing are becoming increasingly critical to educational institutions mainly driven by the advancements in computer technology and the Internet.

Electromechanical energy conversion is a required course usually at junior level in most of the electrical engineering programs. This course typically consists of classroom and 
laboratory sections. The laboratory section includes tests conducted to determine equivalent circuit parameters, losses and performance under various loading conditions of various electric machines. In a traditional laboratory, students perform these experiments using specific equipment and instrumentation under the supervision of a laboratory instructor during the time slots of laboratory sessions. There are several shortcomings to the traditional laboratory, such as: a) the cost and space requirements of the equipment are so high that institution often can't modernize them very often; b) students access to laboratories is restricted only to the laboratory periods; c) students can see usually only the inputs and outputs of the system, the experiments cannot fully backup the understanding or intuitiveness of the physical phenomena and principles illustrating the system functionality; d) only a limited number of tests and laboratory exercises can set and performed; and e) operating under unfamiliar conditions, accidents may happen.

Virtual experiments and laboratory especially in the areas of electric machines and power systems are increasingly used due to several advantages and flexibility. The major advantage of virtual laboratory lies in the possibility of communicating information through high-speed communication systems, when needed, with a local facilitator to supervise the learning process at a local site ${ }^{1,2,10,18-20}$. The local facilitator is a professor at a local university that will use the developed virtual system for instruction. Educators crunched between demand for an effective education and budget constraints, can use virtual instrumentation to automate routine tasks, accomplish new objectives, replace outdated and expensive equipment, and/or to demonstrate students the potential of high technology.

Our electric machines course introduces the students to various types of electric machines, parameters, characteristics, and tests of electric machines, and their applications ${ }^{14-17}$. The main types of electric machines studied during this course are transformers, DC machines, induction motors, synchronous machines, single-phase and special motors. The on-site laboratory consists currently of eight experiments, conducted during the course of a term, by using ElectroVolt educational system. They are: 1) Getting Familiar with the Laboratory; 2) Measuring Impedance and Power; 3) Transformer Excitation, Transformer Equivalent Circuit; 4) DC Generators; 5) DC Motor Load Characteristics; 6) Synchronous Machines; 7) Induction Machines; and 8) Single-Phase Motors.

\section{Virtual Laboratory}

There are several benefits, advantages, as well as drawbacks of the virtual laboratories comparing to the traditional ones. However, this section is not intended to provide a comprehensive coverage of the benefits and drawbacks of the virtual laboratories. In a traditional laboratory students gain an experience as close as possible to real life given the limitations of the laboratory environment. The hands-on' experience often results in much wider student interest than a classroom lecture. This is the most significant advantage of a traditional laboratory. Student access to laboratory rooms is typically restricted to the allotted laboratory hours, and is usually only permitted when a staff member is present. As a result precious laboratory space and expensive equipment is unavailable for student use most of the time. In fact pure logistics are often the main drawback of the traditional laboratory ${ }^{1-6}$.

Virtual laboratories are computer-simulated laboratories that look like, operate as, and produce results similar to real ones ${ }^{4,5,18-20}$. A virtual laboratory is characterized by its versatility and flexibility because it is software based. Like traditional laboratories, virtual 
laboratories have a number of advantages and disadvantages. Overall, in many situations the advantages of a virtual laboratory can outweigh the disadvantages, thus making it a feasible option. Among their advantages can be: a) Cost. As the computer replaces some or all of the laboratory equipment, purchase, maintenance and storage costs are all likely to be reduced ${ }^{6}$. b) Safety. Safety concerns often limit the scope and scale of traditional experiments. A virtual laboratory can either filter out what would be potentially destructive parameter settings in real life, or simulate the damage that would be caused. c) Availability. If a laboratory is fully simulated and available via a browser then it can be performed at any time from anywhere ${ }^{3}$.

d) Presentation of Material. The multimedia capabilities make it possible to present material in a variety of ways, potentially increasing student learning. e) Result Recording. The computers automatically record, process and present measurements, reducing the student procedural overhead, providing more time for useful activities. f) Administration. The computer can easily keep records of the student work, reducing the administrative burden on staff. Some of the disadvantages are: g) Unrealistic. Where the computer completely simulates a piece of equipment students can find the laboratory too far removed from reality to be useful. Real life apparatus always contains an element of unpredictability, which can provide valuable lessons in a laboratory situation. h) Poor Design. Virtual laboratories need to be carefully designed. If not designed properly, computer based laboratories can amplify previous problems and even create new ones ${ }^{4}$. i) Lack of Student Control. In most cases students must complete virtual laboratories in the order dictated by the computer, however, the better designed packages allow students to repeat sections as required. The lack of faceto- face contact means there is little opportunity to discuss ideas or concepts, which fall outside the range covered by the simulation. In many cases it seems as if the ideal solution is, where possible, to combine a virtual laboratory with a traditional ones.

The Electrical Machines Virtual Laboratory proposed and being underway to be developed integrates computers and industrial hardware thus expanding the range of instrumentation and control to virtually unlimited capabilities while offering for the students a pre-practice, a replacement or a post-analysis of the real laboratories. The methodology used in this project is structured in a same manner as in the case of the real lab. It begins with an introduction containing a short discussion on the laboratory exercise to be performed, procedures, analysis, report writing requirements and references.

\section{II.1 The Virtual Interfaces}

As the project outlined in this paper demonstrates, with suitable resources a virtual laboratory can aid students in their preparation for carrying out the experimental work. The virtual interface planned to be developed involves creating simulations of the machine experiments on a website. Students log in, configure parameters and then "perform" the experiment. Simulations return results consistent with the machines in the laboratory. It is envisaged that in this way all students will be able to perform all parts of all experiments in their own time. Students will still attend "hands-on" laboratory sessions to conduct selected parts of the experiments as physical resources permit. The application would be available over a network, and accessible via a web browser. The individual experiments are programmed in LabVIEW and MATLAB/Symulink. Many virtual laboratories require users to install some software on their PCs. This is especially true of those which incorporate tools such as: LabVIEW and MATLAB ${ }^{10-13,21,22}$. An advantage of incorporating all functionality into applets is that no user installation is required, the entire session executes within the web browser environment. When a student selects an experiment from the website the appropriate applet is downloaded 
to their PC and executes locally. The advantage of this approach is that the student is encouraged to repeat parts of the experiment, varying different parameters as required, as there is little downtime between requests.

Virtual instrumentation refers to use computer signal processing, data acquisition, processing and display. In this project we are using a LabVIEW, a National Instrument Software Product. Each LabVIEW program is called a "virtual instrument" or a VI. It consists of two subsystems: a) the actual instrument panel, containing controls (such as buttons, knobs, etc.) and indicators (meters, LEDs, graphs, etc.), and b) the wiring diagram of the VI. First part of the VI is used during the collection and analysis of the experimental data. The front panel for a simple VI is shown in Figure 1. The wiring diagram shows how the data is collected, processed, and routed. But the wiring diagram is not just a visual representation of the computer program: is the program. Programming in LabVIEW is entirely graphical. Many of programming features available in "normal" programming languages, such as: control statements, loops, signal processing, built-in functions, etc. are available in LabVIEW as well. A number of VI's were written for this laboratory (about 20). Many of the VI's are customized to be used in a particular experiment, although about one third of them are general purpose. Some experiments require more than one VI, especially those of the electrical machine tests and performance evaluations. Also, a number of subVI's (subprograms which are themselves virtual instruments) were written.

\section{II.2 Choice of Software}

The current perception among the engineering disciplines is that multimedia tools are appropriate only for the courses which offer very little individualized help for students, and therefore, they are not suitable for engineering subjects which require technology-intensive hands-on experience. However, recent developments and applications, specifically the LabVIEW-based applications, have shown that many pure lecture-based engineering courses and conventional experiments can be updated and integrated with custom-written VI. In addition to this the courses and experiments can be delivered remotely without having multiple copies of the experimental setups. Additional scientific visualization and advanced analysis can also be added, which is limited in the conventional laboratories. Moreover, the VI approach is open to further improvements and developments, which may increase the student participation and enthusiasm while providing an ideal delivery environment. A number of interactive computer-delivered simulation, control, and scientific visualization software solutions are available in the market, and many application-specific tools have already been reported in the literature ${ }^{1-3,7,18,19}$, which use diverse software, such as Hypertext, Authorware, Director, Labtech, Visual C, Visual Basic, MATLAB/Simulink, and LabVIEW. It is found that the following criteria may be contemplated ${ }^{10}$ for selecting application software to build virtual instrumentation used in engineering education:

a. Modularity, allows to test individual modules easily and to develop applications quickly. b. Multi-platform portability enables designers to work on separate parts and compile them on one platform.

c. Compatibility with existing code allows incorporating with previous applications, and also with the previous versions of the software.

d. Compatibility with hardware allows the ability to gather data from different interface hardware. 
e. Extendable libraries allow the designer to build libraries of low-level routines to link them in higher level systems.

f. Advanced debugging features to optimize product design and debugging.

g. Executables, to avoid alteration, to hide the code or to create standalone applications.

$\mathrm{h}$. Performance, to ensure that the end product meets the required performance.

i. Intuitive Graphical User Interface (GUI), enables a user to look at it and see what needs

to be done

j. Multimedia capabilities, for future developments.

The ideal software tool should have all the features listed above. However, the commercial packages available in the market for the engineering courses are either very complex to use or do not provide all the necessary functions to achieve the certain tasks, or are too expensive. Because of its overall versatility as an engineering tool, the software package LabVIEW was chosen in this project. Furthermore, the software meets most of the software selection criteria, and it not only does the data manipulation, analysis, and control, but also has some multimedia authoring capabilities with the help of the add-on tools. The LabVIEW software allows for the creating of application-specific templates to reduce the production time for the identical subjects. Many useful functions can be incorporated with the LabVIEW programs to perform very useful tasks in a laboratory virtual instrumentation system design. Some of these useful functions ${ }^{9}$ are listed below: a) adding warning messages; $b$ ) providing instructions, pre-practical tutorials and/or interactive tests; c) generating a test report or a data file in a text format; d) printing charts or the user front panels; e) linking to other available systems or software; f) inclusion of passwords to limit the access; g) animating the system operation; h) providing GUI that mimics the real instruments; and, i) playing a video ${ }^{10-13}$.

Figure 1 illustrates a sample front panel of LabVIEW virtual instrument, and a sample block diagram that shows the simulation of a motor control drive in LabVIEW. As can be seen in Fig. $1 b$, the modularity of LabVIEW programming allows the designer to simplify the programming structure by using sub-virtual instruments, which may require thousands of lines of codes in other programming software tools. The full-development LabVIEW software comes with a number of additional tools that provide some exceptional features. The Internet Developers Toolkit is a collection of libraries for converting virtual instruments into Internet-enabled applications, and offers unique and innovative distance learning solutions. The projects created by LabVIEW can be converted into standalone applications by using the Application Builder Toolkit, which does not require a copy of LabVIEW to run. One of the features of the LabVIEW programming is the hierarchical nature of VIs. Once a VI is created, it can be used as a sub-VI in the block diagram of a higher level VI. The LabVIEW seems one of the primary choices in designing control and analysis solutions in the area of engineering and education. One of the advantages of the most National Instruments boards is this that they can be also used with the MATLAB software.

\section{LabVIEW Applications as an Educational Tools for Electric Machines}

In our study of electrical machines, several computer simulations have been developed or are underway to be developed to get a full set of electric machine experiments, as discussed in section II of this paper. However, many others agree that simulation is not a substitute for

actual experiments ${ }^{4}$. This paper will emphasize some of the capabilities of utilizing virtual instrumentation to study transformers, DC machines, and synchronous machines, already 
developed and implemented. A laboratory management support system is planned to be developed in the near future.

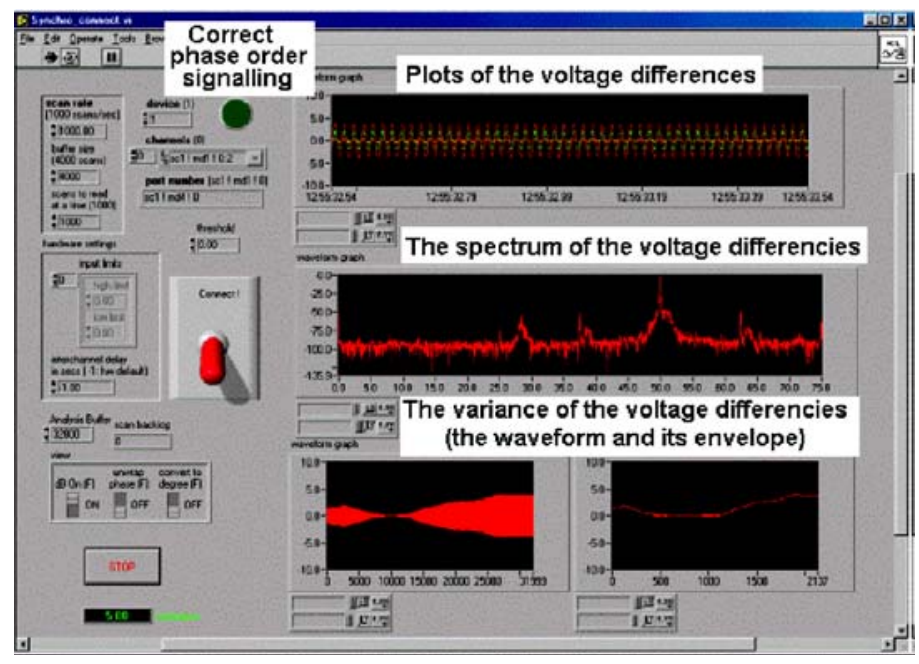

a)

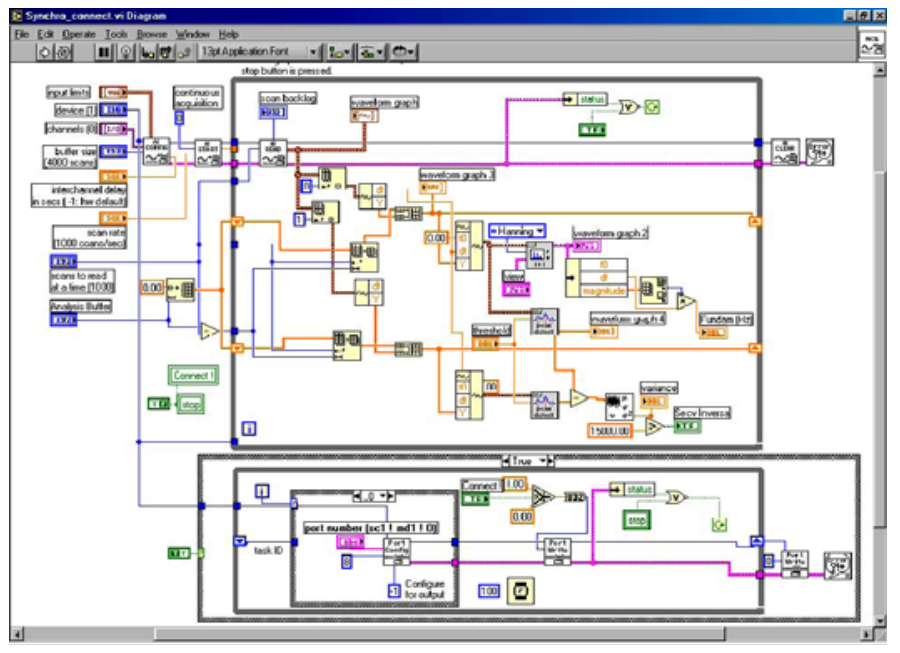

b)

Figure 1: a) The main front panel and b) the block diagram of AN Vi.

\section{III.1 Single-phase Transformers}

To study the characteristics of a single-phase transformer voltage is applied to the primary of a single-phase two-winding transformer. In the transformer excitation experiment, the voltage is applied to the primary winding of a single-phase two-winding transformer. The primary current is measured as is the secondary voltage with no load present at the secondary. The socalled exact equivalent circuit of such a transformer is shown in Figure 2. This equivalent circuit neglects the nonlinear aspects of the core loss, etc. For small input voltages, the relation between flux and exciting current is linear. Using the VI designed for this experiment the hysteresis curve obtained is shown in Figure 3. 


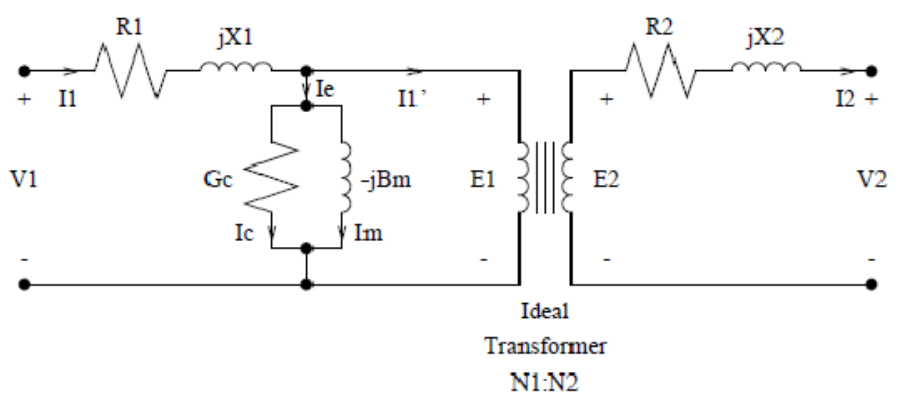

Figure 2: Equivalent circuit of a transformer

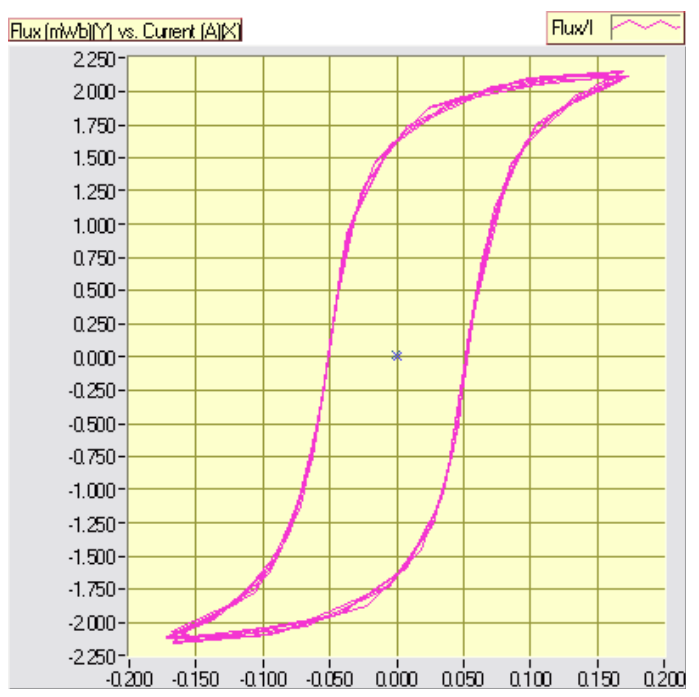

Figure 3: Hysteresis curve of a single-phase transformer.

\section{III.2 DC Generator Characteristics}

One of the VIs developed makes it very convenient to build a graph of one variable versus another variable. Students can record the data and plot all the corresponding points on the graph. Moreover, making multiple graphs on the same set of axes is possible, which is very useful when comparing characteristics of various machines. If required the students can delete an entire graph or multiple graphs at once. This application is used to plot the performance characteristics of various electrical machines. For example, the VI can be used to plot the magnetization curve of a DC generator, as shown in Figure 4. This is a single graph of armature voltage versus field current at a constant speed. This curve corresponds to the magnetization equation $E_{a}=K_{a} \phi_{p} \omega_{m}$, where $\mathrm{E}_{\mathrm{a}}$ is the generated voltage, $\mathrm{K}_{\mathrm{a}}$ is the machine constant, $\omega_{\mathrm{m}}$ is angular velocity, and $\phi_{\mathrm{p}}$ is the flux per pole.

The characteristics of different DC generators in loaded condition are shown in Figure 5, which depicts a set of 3 graphs of load voltage versus armature current. The top-most curve represents a separately excited DC generator. The curve in center represents a cumulatively compounded long shunt DC generator and the third curve represents a self-excited DC 
generator. As expected, the separately excited DC generator has drooping characteristics because of the losses due to armature resistance, brush contact and armature reaction. Whereas, in the case of the self-excited shunt DC generator the field excitation is provided by the generator itself so reduced terminal voltage will supply a reduced field current which causes further reduction in the voltage. In a cumulatively compounded generator the series and the shunt fields aid one another. As the load current increases, the current through series winding increases, therefore, field strength increases compensating the decrease in shunt field strength. As a result, the overall magnetic field strength increases, which is the cause of increase in the output voltage of the generator as compared to that of the self-excited case.

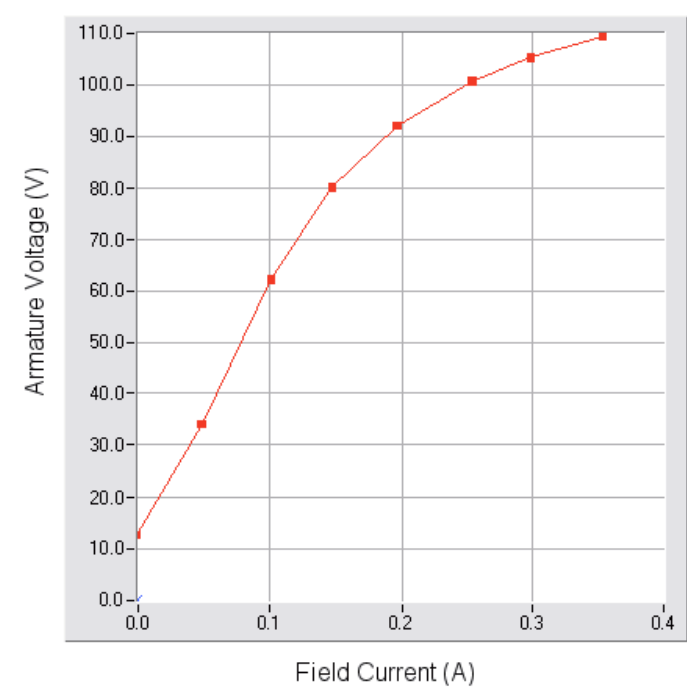

Figure 4: Saturation Curve of DC generator.

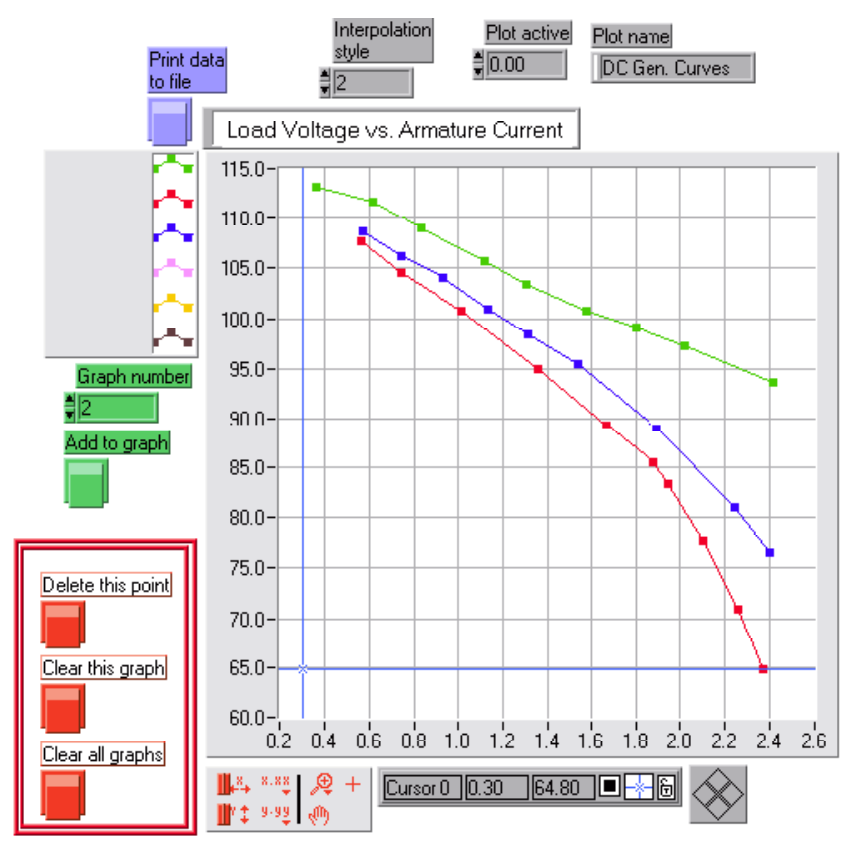

Figure 5: DC generator performance. 


\section{III.3 Synchronous Machine}

Synchronous generator simulation is straightforward once the fundamental features of LabVIEW software are mastered. In electrical generator, the mechanical energy input and the electrical energy output can be presented in mathematical form, after presenting the physical operation of the generator with the equivalent electric circuit of the 3- $\phi$ synchronous generator ${ }^{15}$. The electric circuit is used to facilitate the calculation of the unknown quantity, for instance current or voltage, once the values of the resistive and inductive components are given. The values could be evaluated experimentally by conducting short-circuit and opencircuit tests on the generator if that is possible; otherwise, the manufacturer should be contacted for the information. The power flow, within the generator is tracked by balancing the input and the output taking into account the heat and magnetic power losses. The losses are quantified by performing several standard tests on the generator. The steady-state developed torque and power are then evaluated and plotted to reveal the generator characteristics. The expected efficiency of those particular parameters can also be plotted. Almost every textbook presents the synchronous generator by per phase equivalent circuit (Figure 6), and shows how steady state current and power are estimated. In many cases, the armature resistance and copper losses are ignored to simplify the procedures. In many textbook examples ${ }^{14-17}$, the synchronous generator current can be estimated with fairly acceptable accuracy using the above methodology presents the standard steps to determine the internal generated voltage using the equivalent circuit for the synchronous generator.

The practicality of using LabVIEW software for the student is that it will be possible to input various configurations of variables without any knowledge of text-based programming. The use of the built-in functions of the software in an interactive way to produce the complete generator characteristics over the entire variable range instead of just one operating point will be more informative for the student. This is one of the advantages over the numerical examples normally presented in the textbook. Therefore, the student can verify all the possible operating points along the generator characteristics. The LabVIEW simulations stored for the student in the database of the course generate complete characteristics over the whole variable range allowing the student to examine the shape and verify different operating points. Furthermore, the software can be used by the students for verifying laboratory experiments after entering the laboratory generator data and the operating conditions. The recorded test results for the laboratory machines could be compared for further verification between theory and practice. To investigate the complete generator characteristics under varying conditions, several cases are simulated, such as ones presented in the next paragraph.

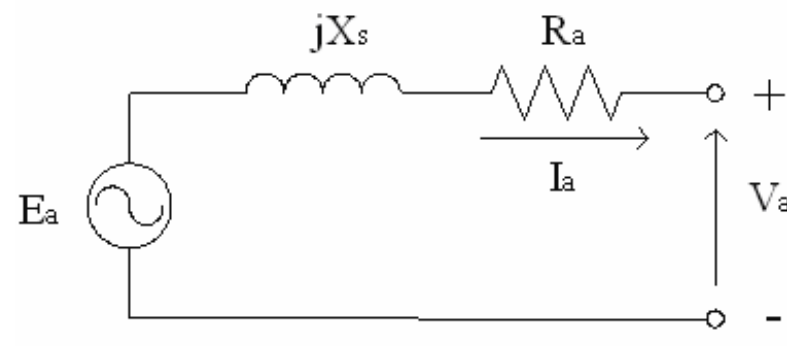

Figure 6: The per-phase equivalent circuit of the synchronous generator 
Output Power: Case 1: Effect of torque angle on output power: Output power varies sinusoidally with the torque angle if the phase voltage and internal generated voltage are assumed constant (Figure 7). Case 2: Effect of synchronous reactance on output power: Output power varies inversely with the synchronous reactance if the internal generated voltage and phase voltage are assumed constant, as shown in Figure 8. Case 3: Effect of internal generated voltage on output power: Output power varies directly with the internal generated voltage if the phase voltage is assumed constant (Figure 9).

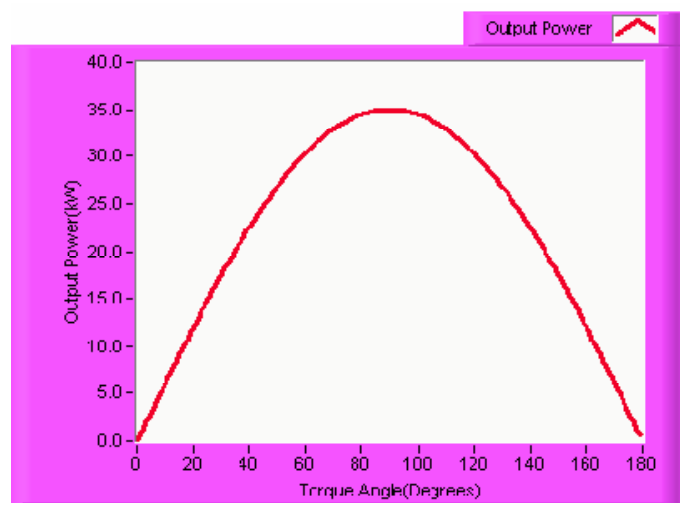

Figure 7: Graph generated in LabVIEW showing the variation of output power with the torque angle.

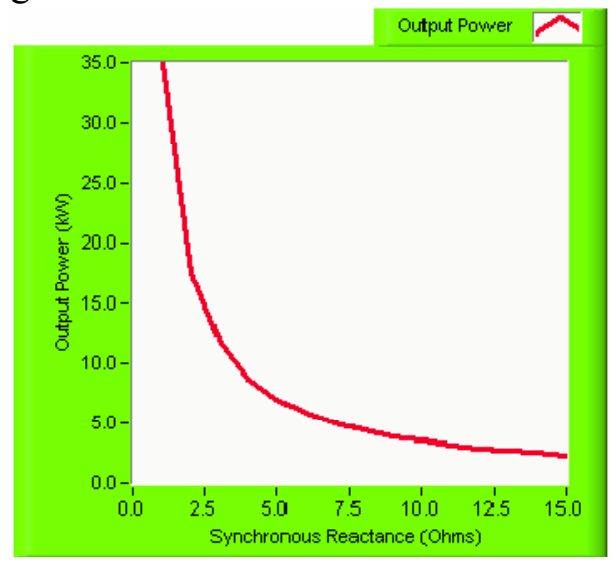

Figure 8: Graph generated in LabVIEW showing the variation of output power with the synchronous reactance.

\section{Conclusions and Discussions}

The techniques and capabilities of an improved laboratory practice have developed and improved undergraduate experience and learning for the students in our program. The Internet now offers new non-traditional possibilities in order to improve and complete the classical educational methods. Hence, the presented laboratory work will be extended in a manner to be accessed also from distance using the Internet. Electrical engineering students can use the Electrical Machines Virtual Laboratory in order to prepare themselves for the real laboratory exercises. The purpose of these experimental setups in association with the appropriate LabVIEW and MATLAB software packages presented in this paper is especially designated to be used for testing of electrical machines. The use of virtual instrumentation 
has not only provided a modern and interesting way for students to perform experiments but also has reduced the amount of time necessary to make connections. The real-time display of quantities have made great improvements in the students' ability to visualize these quantities and understand their relation to one another. The simulation feature which is included in the main LabVIEW program is also useful for the student's practices. Meanwhile the students will have access to files containing information on the basic principles of the measuring method, the basic parameters of the system in study, and will also have access to files where the students can change the parameters and watch how the system's behavior is influenced.

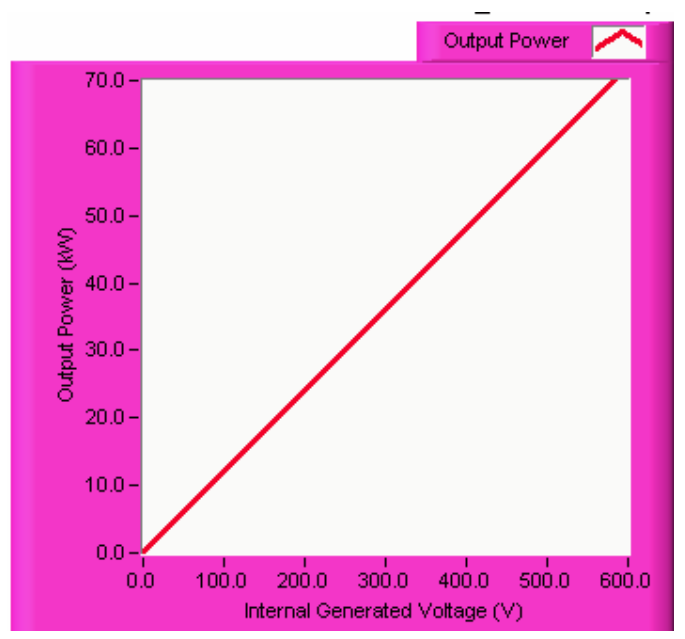

Figure 9: Graph generated in LabVIEW showing the variation of output power with the internal generated voltage

In the complete development, each of the virtual laboratory modules will also contain an assessment of the work. Each of them will contain several different questions bearing on the discussed topics: electrical machine's principles, parameters that may be used in simulations, or the basic properties of the measuring method. For each question a single correct answer has to be selected from a set of possible answers. There are the possibilities in the continuous develop of the software application that would allow it to calculate and display various parameters.

\section{References}

1. Ertugrul, N., (1998). " New Era in Engineering Experiments: An Integrated Interactive Teaching/ Learning Approach and Real Time Visualisations", International Journal of Engineering Education, Vol.14, No.5, pp. 344-355..

2. Ertugrul, N., A. P. Parker, and M. J. Gibbard, (1997). "Interactive Computer-Based Electrical Machines and Drives Tests in the Undergraduate Laboratory at The University of Adelaide", EPE'97, 7th European Conference on Power Electronics and Application, Trondheim, Norway, 8-10 September 1997.

3. G. Bengu and W. Swart, A computer aided, total quality approach to manufacturing education in engineering, IEEE Trans. Educ., vol. 39, Aug. 1996.

4. M.W.Daniels and R.A.Shaffer. Re-inventing the electrical machines curriculum. IEEE Transactions on Education, 41(2):92-100, May 1998. 
5. Thomas W. Gedra. Computer-aided instrumentation in OSU's machines lab. In Proceedings of the Frontiers of Power Conference, pages VIII.1-VIII.5, Oklahoma State University, Stillwater, OK, October 1997.

6. Thomas W. Gedra. Virtual instrumentation in an undergraduate electrical machines lab. In Proceedings of the Midwest Section ASEE Conference, Columbia, MO, April 1997

7. G. Faraco and L. Gabriele, Using LabVIEW for applying mathematical models in representing phenomena, Computers \& Education, Volume 49, Issue 3, November 2007.

8. C. Elliott, V. Vijayakumar, W. Zink and R. Hansen, National Instruments LabVIEW: A

Programming Environment for Laboratory Automation and Measurement, Journal of the Association for Laboratory Automation, Volume 12, Issue 1, February 2007.

9. R. Krishnan, A. Bharadwaj, and P. Materu, Computer aided design of Electrical machine for variable speed applications, IEEE Trans. Ind.Electron., vol. 35, no. 4, Nov. 1988.

10. S. Linke, J. Torgeson, and J. Au, An interactive computer-graphics program to aid instruction in electric machinery, IEEE Comput. Applicat.Power, July 1989.

11. N. Kehtarnavaz and N. Kim, LabVIEW Programming Environment, Digital Signal Processing System-Level Design Using LabVIEW, 2005.

12. N. Kehtarnavaz and N. Kim, Getting Familiar with LabVIEW: Part I, Digital Signal Processing System-Level Design Using LabVIEW, 2005.

13. N. Kehtarnavaz and N. Kim, Getting Familiar with LabVIEW: Part II, Digital Signal Processing System-Level Design Using LabVIEW, 2005.

14. S.J. Chapman, Electric Machinery Fundamentals, McGraw-Hill, 2005.

15. A. E. Fitzgerald and C. Kingsley, Electric Machinery, McGraw-Hill, 1992.

16. G. Mcpherson and R.D. Laramore, An Introduction to Electrical Machines and Transformers, New York: John Wiley.

17. A. Nasar, Electric Machines and Power Systems, Volume 1, Electric Machines, Tata McGrawHill, 2002.

18. I. Gustavsson, "A Remote Access Laboratory for Electrical Circuits Experiments," International Journal on Engineering Education, vol. 19 (special issue on Distance, Controlled

Laboratories and Learning Systems), no. 3, pp. 409-419, 2003.

19. P. Grätz, and S. Šedivá, "Virtual Laboratory of Sensors and Measurement of Non-electrical Quantities," CEEPUS Cz-103 International Summer School ACTA '2003.

20. Saliah, H.H., Abecassis, A., Nurse, E., "Design of a Generic, Interactive, Virtual and Remote Electrical Engineering Laboratory," Proceedings of the 29th ASEE/IEEE Frontiers in Education, San Juan (Puerto Rico), pp. 255-262, 1999.

21. "LabVIEW User Manual," National Instruments, Austin, 2000.

22. Gillet D. et al., "Remote Manipulation with LabVIEW for Educational Purposes," in: Internet Applications in LabVIEW, Prentice Hall PTR, Upper Saddle River, 2000. 